\title{
Industry Regulation through Open Source Software: A Strategic Ownership Proposal
}

\author{
Jean-Lucien Hardy \\ Eurocontrol \\ jl.hardy@eurocontrol.int
}

\begin{abstract}
This paper is about a twofold proposal submitted to the scrutiny of the OSS scientific community. It is first argued that OSS should be considered a means to establish an industry regulation. The motivation of this first proposal is the need for harmonization of the supply chain in certain industrial sectors. The Air Traffic Management industry (ATM) is the only case considered in this paper. However, it is assumed that the regulatory advantage of OSS is not specific to that industry. The second proposal is about how to establish such a regulation through OSS. It is argued that the legal ownership of the OSS product should be assigned to a public organization, preferably to an organization that would be dedicated to monitor and promote the evolution of that product. The motivation for these proposals is based on the analysis of possible scenarios of OSS ownership in the case of ATM. Perspectives concerning the preliminary implementation of the proposals are introduced.
\end{abstract}

Keywords: Industry Regulation, Open Source Software, OSS, Intellectual Property Rights, IPR, Ownership, Secondary Software Sector, Air Traffic, ATM, ATC.

\section{Introduction}

In this introduction, the role of the scientific community in OSS adoption will first be discussed. Second, the strategic importance of legal ownership of OSS products is emphasized. Third, the absence of a public regulatory role concerning OSS is pointed out. Fourth, the context of ATM is introduced as a target case.

\subsection{The Role of the OSS Scientific Community}

Science is built on research results, but can only progress with development and foresight. The role of the scientific community is not only to do research on existing phenomena, but also to advise decision makers about appropriate deployment choices, based on clear concepts and hypotheses. OSS is not a fundamental science. Like the global warming issue, OSS has a strong social component and depends heavily on managerial or political decisions. The role of the scientific community is critical in terms of advices to decision makers involved in long term planning. A scientific approach must guide the decisions concerning the strategic use of OSS. 
Scientific advisers should discuss their advices first within the scientific community, before presenting them to decision makers. Such an open work-flow is particularly important when the domain is related to public issues and public governance. OSS is such a domain intrinsically connected to public issues.

A scientific conference about OSS is an opportunity to emphasize, discuss, refine and publish hypothetical scenarios for decision makers, in order to focus further research on concepts and hypotheses useful beyond the academic world.

\subsection{The Ownership of OSS Products}

In legal discussions concerning the adoption of OSS, there is generally more emphasis on the choice of an appropriate license than on the choice of appropriate ownership of IPR (Intellectual Property Rights). However, the choice of ownership is a prerequisite to the choice of an appropriate license.

Informal discussions with people unfamiliar with the OSS domain tend to show that many of them are aware of a special kind of software license for OSS without understanding that there is a legal owner behind this license. The word "public" in the name of the most popular OSS license (GPL stands for "General Public License") contributes to such a misunderstanding of which the consequences are numerous. First, it must be counter-explained to people who discover OSS that a product distributed under GPL terms does not belong to the public. Second, the unnecessary multiplication of OSS licenses may well be motivated by the hidden pride to emphasize the existence of a product owner erroneously considered "public". Third, the perceived lack of an OSS owner who could be held responsible or accountable for problems is used as an excuse for not adopting OSS products [1].

The power of the IPR owner is critical in terms of business, as shown by the case of MySQL and OpenOffice.org, two popular OSS products whose ownership was recently transferred as an asset through the acquisition of Sun by Oracle. Oracle Corporation is now in a strong position to promote or jeopardize the OSS spirit concerning these products. There is actually no legal obligation for Oracle to consider the public interest in its strategy and support to OpenOffice.org and MySQL. Hopefully, it will be to its corporate and commercial advantage to promote the public interest.

In this paper, it is argued that the ownership of OSS products by public organizations could be the leverage of a new regulatory role.

\subsection{The Absence of a Public Regulatory Role Concerning OSS}

In terms of IPR, there are tremendous regulatory efforts built around legal patents. This regulation is transcribed into legal procedures administered by strong public organizations (patent offices). However, there is nothing similar for OSS. A quick look at the literature confirms the absence of a governmental regulatory role concerning OSS. The word "regulation" is not mentioned in any title of the hundreds of papers presented to the previous IFIP conferences on OSS. The interaction between industry regulation and OSS does not seem to have yet been investigated.

In this paper, it is argued that regulation of an industry through OSS could be effective in the interest of all players in that industry, and first of all the customers. 


\subsection{The ATM Supply Chain}

Air Traffic Management covers a spectrum of activities including real-time Air Traffic Control (ATC), planning air traffic flow, design of 3D routes for air traffic, environmental concerns in air traffic, taxation of air traffic, etc.

From a conceptual point of view, it has been recently pointed out in a research panel [2], that an ATM supply chain exists, organized on a continental basis. In Europe, the ATM supply chain includes:

- the so-called "ATM industry" selling the ATM systems,

- the so-called ANSPs (Air Navigation Service Providers) mainly providing ATC services,

- many ATM sub-contracting firms supplying particular hardware and software equipment, and

- the public administrations (civil and military) contributing to operations, research, co-ordination, regulation and taxation. EUROCONTROL is such a public organization at the European level.

Most ATM activities are software intensive. Software tools are used for operations, as well as for research and innovation. In terms of software categorization, ATM is part of the secondary software sector [1,3]. Most ATM software is currently proprietary.

In this paper, the ATM supply chain is considered a study case for a new kind of regulation based on OSS.

\section{Failure of OSS Adoption in ATM}

There are no formal research results about the adoption of OSS in ATM. However, from the beginning of this century there were some serious initiatives by EUROCONTROL to increase awareness and to study the potential of OSS in ATM [4,5,6]. ATM was also considered a case study in the European CALIBRE project. Subsequently, the author was interviewed in the context of a formal academic research concerning the adoption of OSS in the secondary software sector [1].

A general consensus concerning the potential of OSS in ATM emerged from various events and conferences. One of the main arguments is that OSS could help harmonize ATM systems in Europe. For the last two decades, the need for this harmonization has been frequently expressed. Recently, some thorough performance studies have highlighted the important costs of fragmentation of the ATM solutions in Europe [7].

Considering the concept of an ATM supply chain [2,8], OSS appears to be the right harmonizing technology, as it improves software co-operation and interoperability.

When he visited EUROCONTROL for a first seminar in October 2009, Rishab Ghosh also emphasized benefits in terms of sustainability [9], since OSS offers better guarantees than proprietary software, especially in a niche market like ATM. Sustainability seems to be necessary to guarantee harmonization over time.

Other technical and business benefits, as well as drawbacks, have been reported for the European secondary software sector [1,3]. Ideally, there should be a systematic research in terms of Pareto analysis to determine what the most critical drawback is. However, this research could hardly be based on strong facts, but rather on statistics 
based on experts' opinions that would be artificially consolidated for the sake of a paper. In the present paper, a Pareto analysis is based on rational reasoning built on top of the study by Lorraine Morgan and Patrick Finnegan [1], and applied specifically to the ATM case.

This study reports that the business drawbacks appeared to pose a bigger challenge for OSS than their technical counterparts. The lack of backing support from the company and the lack of ownership are mentioned first. The other business drawbacks found in the same study seem to be derived from the lack of backing: insufficient marketing, training, competencies and access to code.

In terms of top decision making for the ATM domain, it seems that the harmonization and sustainability benefits expected from OSS in ATM cannot explain the lack of managerial backing.

Therefore, the hypothesis considered in the present paper is that the lack of relevant ownership is the most critical drawback from which the others are derived, including the lack of backing support. In terms of a Pareto analysis, it is useful to guide the decision making with a proposal to overcome this major drawback.

\section{Seven Counter-Productive Scenarios for OSS Ownership}

Various scenarios of OSS adoption can be considered depending on who is the IPR owner of the OSS product(s). Considering the classical limiting factor analyzed by G.A. Miller [10], seven scenarios are discussed here concerning ATM.

\subsection{OSS Owned by a Small Company}

It is possible for a small company that owns a software product to publish it in OSS mode, inviting anyone to join a community to develop and improve that product. There was a recent example of such a scenario in ATM (www.albatross.aero). For a profit oriented company, the ownership can be motivated by financial speculation on the value of the IPR enhanced by the value of the OSS community that develops the OSS product. However, such an underlying speculative objective could hardly serve the efficiency of the ATM supply chain, because it cannot be shared by other partners. Therefore, an OSS tool driven by financial speculation would probably increase fragmentation in ATM.

\subsection{OSS Owned by a Major Player in the Industry}

In May 2006, there was a CALIBRE meeting in Spain where two major telecommunication players, Vodafone and Telefonica, explained their OSS initiatives: since the differentiating power of the software was rather low - Telefonica said 5\% they decided to go open source in order to enlarge their supplier basis. It appears that the two companies were creating their own OSS projects, as neither of them expressed a willingness to join the OSS initiative of the other. Telefonica and Vodafone gave the impression of using OSS to compete in attracting more suppliers.

If the same scenario happens between major ATM companies, it would not be a solution for the whole ATM supply chain, because the duplication of the OSS community would not contribute to harmonization. 


\subsection{OSS Owned by an OSS Foundation}

The copyright of the ADA development environment GNAT was created by a grant from the US Air Force to the New York University. The copyright of GNAT was assigned from the New York University to the Free Software Foundation (FSF). Such a scenario prevents speculation and favors fair competition [11]. Now, what if the IPR of ATM tools were owned by an OSS foundation, like the FSF? Such a scenario would probably not work well, because the ATM domain is a specific niche, and because the FSF is not part of that niche. Therefore, such a foundation could not manage the use of the OSS ownership in the interest of the ATM world. For example, if there were a demand for a commercial license, a disconnected OSS foundation would have neither the means nor the competence to negotiate this demand for the benefit of the ATM niche and its specialized players.

\subsection{OSS Owned by Cooperatives of Users}

Some ATM software tools have their own informal community of users. It could be easy to create ad hoc legal bodies on top of these communities and to assign them the OSS copyright. The drawback of such a scenario would be the addition of an administrative layer with a need for co-ordination, and therefore yet another source of fragmentation in the ATM sector. Given the volatility of such small legal bodies, the sustainability criteria would not be fulfilled either.

\subsection{OSS Owned by a National Public Administration}

A country in EUROPE could decide to use OSS for its ATM sector. It would make sense, especially for countries that have a long tradition in public administration of ATM. This would facilitate fair procurement for the development and maintenance of OSS tools while avoiding the risk of speculation. However, such a national scenario would reinforce the existing geographical fragmentation of the European ATM.

\subsection{OSS Owned by a Continental Public Organization}

EUROCONTROL, a leading public organization in European ATM, is the owner of a portfolio of software tools used for either operations or R\&D (Research and Development). Recently, there were two attempts to publish R\&D tools in OSS terms (nogozone.sourceforge .net and atv3d.sourcegorge.net). However, such a publication cannot be extrapolated for commercial software tools, because EUROCONTROL is a public body that is not supposed to act in the role of a Pan European software house disturbing competition in the ATM market.

\subsection{OSS Owned by a Global Public Organization}

ATM is also in the scope of worldwide organizations. ICAO is a specialized organization of the UN dealing with civil aviation. IATA and CANSO are trade organizations for airlines and ANSPs. Assigning the OSS ownership to such bodies would have the advantage of providing global harmonization. However, performance studies indicate that the improvement needs are not the same in Europe and the US [7]. Therefore an attempt to reach a global harmonization through OSS tools is not a relevant scenario. It seems useful to keep different ATM solutions based on continental specificities. 


\section{Attaching the OSS Ownership to an Industry Regulatory Role}

The seven scenarios of OSS ownership are not productive because the owner does not have a role that justifies its ownership on behalf of the OSS community.

Therefore, the question is whether it is possible to find such an appropriate role. From the seven scenarios, it appears that such a role must include protection against speculative or competitive use of OSS ownership, and governance in the niche domain of the OSS tools for the best interest of the public, e.g. aircraft passengers.

Considering that European ATM needs harmonization (and not fragmentation) of its supply chain, a regulatory role seems to be needed [8]. Since OSS technology has intrinsic advantages in establishing harmonization, the OSS ownership appears to be a prerequisite or an advantage for the creation of a regulatory role based on OSS.

Such a role must be assigned to a public organization at the level of the situation to be improved and harmonized. To tackle the fragmentation of the European ATM, the European Commission and EUROCONTROL are two candidate institutions, but EUROCONTROL is probably better suited for a specific OSS role in ATM given its mission that covers technical aspects of ATM.

To promote its regulatory role, the organization should not be involved in competitive activities such as software development or software services. These activities belong to the private companies involved in the regulated supply chain.

By holding the IPR ownership, a public regulatory body avoids speculative or competitive counter-productive effects. It could also tune the licensing scheme towards harmonization. For example, a dual licensing scheme could be used on demand. The reply would be a regulatory process which allows integration of OSS products with proprietary products. In such a process, the productivity of the supply chain would be optimized in terms of quality improvements and/or in terms of royalties for the OSS community. A preliminary step towards such a dual licensing scheme is implemented by the two ATM tools launched in OSS. For these R\&D tools, the door is left open for a commercial license with a counterpart for the OSS community (nogozone.sourceforge .net and atv3d .sourceforge.net).

Figure 1 summarizes the hypothetical proposal. The harmonization and sustainability need of the whole industry determines the choice of technology (OSS) and the main attribute of the regulatory role (IPR). A key issue is that the regulatory role is also impacted by the choice of technology. Therefore, technology and regulation have a positive and correlated impact on the efficiency of supply chain.

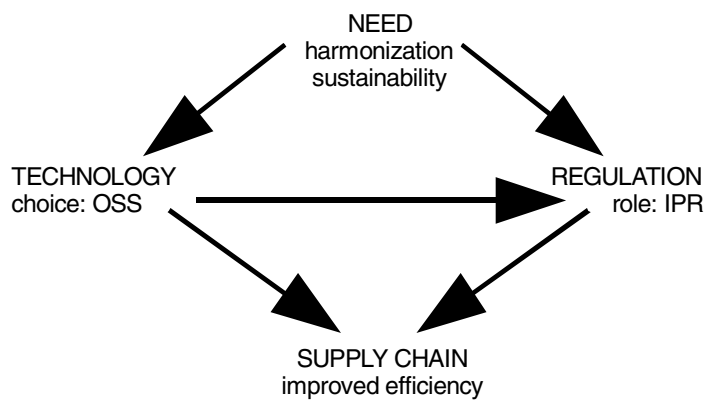

Fig. 1. Technology and regulation contributing to the efficiency of the supply chain 


\section{Conclusion and Future Perspectives}

This paper describes a twofold strategic proposal. First, it is argued that OSS should be used as a tool for establishing industry regulation when needed. In particular, such a need exists in the case of a complex industrial supply chain using software products: the regulated approach through OSS is a way to achieve sustainable harmonization. The second argument is that an industry regulation through OSS is possible when the ownership of the OSS products is assigned to the regulatory body, preferably an organization with the necessary domain specific background to monitor and guide the evolution of the OSS products in the interest of the public.

In the particular case of the ATM sector in Europe, EUROCONTROL seems to be in the right position to play such a regulatory role. In a paper concerning the regulation of European ATM [8], Hervé Dumez and Alain Jeunemaître point out "the need to create new tools for thinking European regional regulation and European infrastructure management." The present proposal of an ATM regulatory role through OSS could be an attempt to fulfill this need.

By extrapolation from the ATM case, the proposal could go a step further towards public OSS. The ownership of OSS should be placed in the hands of public regulatory bodies, especially when the need for harmonization becomes greater than the need for innovation. Private companies that own a software product in the phase of commoditization and no longer commercially differentiating [12] should not just offer an OSS license for their product. They could consider donating or selling the ownership of that software product to a public entity in order to facilitate de facto standardization around that product. On the contrary, the ownership of a software tool by a private company, even in the case of an OSS product, is the evidence of a competitive advantage that inevitably hampers contributions, co-operation and harmonization around that tool.

In the end, the validity of the hypothetical strategic proposal will only be established if it is adopted by top decision makers who manage private or public organizations. Based on the present proposal, they could immediately apply the principle of Alan Kay: "The best way to predict the future is to invent it." However, the best approach for the interest of a large public might be in a continuous back-and-forth between innovation and research.

Therefore, some preliminary steps would be useful before a decision can be made concerning the implementation of industry regulation through OSS. First, it would be useful to survey the OSS ownership and to hear the opinions of OSS specialists, in order to predict if the regulation hypothesis is valid a priori, how it should be implemented, and how its value (efficiency and effectiveness) should be evaluated a posteriori. Second, since industry regulation seems to be an innovative matter in the OSS arena, there is a need for case studies. The ATM context might be considered a case study from which lessons could be extrapolated by the OSS scientific community to other industries. For example, in terms of preliminary experiments, a few software products could be used for the experimentation of a regulatory role by EUROCONTROL, with the intention of finding precise legal, technical, economical, social, organizational, and institutional implications of this regulatory role.

It is the role of the scientific community which analyzes OSS phenomena and communities to study the potential of OSS for industry regulation purposes, based on conceptual modeling, surveys, and case studies. The technical and business benefits and drawbacks of the regulatory scenarios should be anticipated to pave the way for future OSS policies and decisions. 
Disclaimer and Acknowledgment. The author is a civil servant of EUROCONTROL, but the present proposal is a personal viewpoint that does not reflect the official views and policies of EUROCONTROL on the matter. The author is also contributing to the OSS2010 conference as a founding member of the IFIP Working Group 2.13 on Open Source Software. He is grateful to the two anonymous OSS2010 reviewers for their challenging comments on the first version of this paper, to his friend John Seifarth of Words-and-Wires-sprl (www.waw.be) for keen comments on the final version, and to his wife Jung Yeon Kim for correcting English mistakes despite an imminent extension of the family. A special dedication goes to Professor Dieudonné Leclercq of the University of Liège who introduced the passion for software into my life in 1975.

\section{References}

1. Morgan, L., Finnegan, P.: Benefits and Drawbacks of Open Source Software: an Exploratory Study of Secondary Software Firms. In: Feller, J., Fitzgerald, B., Scacchi, W., Sillitti, A. (eds.) Open Source Development, Adoption and Innovation. IFIP, vol. 234, pp. $307-$ 312. Springer, Boston (2007)

2. Jeunemaître, A.: Panel "Innovation in ATM". In: EUROCONTROL 8th Innovative Workshop and Exhibition (2009)

3. Ågerfalk, P.J., Deverell, A., Fitzgerald, A., Morgan, L.: Assessing the Role of Open Source Software in the European Secondary Software Sector: A Voice from Industry. In: Scotto, M., Succi, G. (eds.) First International Conference on Open Source Systems (OSS 2005), pp. 82-87 (2005)

4. Hardy, J.-L., Bourgois, M.: Open Source Implications for EUROCONTROL (OSIFE). In: 3rd EUROCONTROL Innovative Research Workshop (2004)

5. Bourgois, M., Hardy, J.-L., O’Flaherty, J., Seifarth, J. (eds.): Potential of OSS in ATM (2005), http: / / www oss-in-atm. info

6. Hardy, J.-L., Bourgois, M.: Exploring the potential of OSS in Air Traffic Management. In: Darniani, E., Fitzgerald, B., Scacchi, W., Scotto, M., Succi, G. (eds.) Open Source Systems. IFIP, vol. 203, pp. 173-179. Springer, Boston (2006)

7. EUROCONTROL and FAA: U.S./Europe Comparison of ATM-related Operational Performance (2009),

http: //www. eurocontrol.int/prc/gallery/content/public/Docs/

US_Europe_comparison_of_ATM_related_operational_

performance.pdf

8. Dumez, H., Jeunemaittre, A.: Restructuring Regulation in Europe. The Case of Air Traffic Services. Concurrences 1, 1-3 (2008)

9. Ghosh, R.A.: Free Software: How Does it Work. In: European Broadcasting Union Semi$\operatorname{nar}(2007)$,

http: //www.slideshare.net/kamaelian/free-software-how-doesit-work

10. Miller, G.A.: The magical number seven, plus or minus two: Some limits on our capacity for processing information. Psychological Review 63(2), 81-97 (1956)

11. Gasperoni, F.: COTS, FLOSS, and Market Freedom in Safety-Centric Industries. In: [5] (2005)

12. Van der Linden, F., Lundell, B., Marttiin, P.: Commodification of Industrial Software: A Case for Open Source. IEEE Software 26(4), 77-83 (2009) 